\title{
Position of the mental foramen in a Moroccan population: A radiographic study
}

\author{
Ahmed Chkoura, Wafaa El Wady \\ Department of Oral Surgery, Faculty of Dentistry, University of Mohammed V Souissi, Rabat, Morocco
}

\section{ABSTRACT}

Purpose: This study was performed to determine the position of the mental foramen relative to the apices of the teeth based on panoramic radiographs in a Moroccan population. We also analyzed gender differences and the symmetry of location within individuals.

Materials and Methods: Seven hundred ninety-four panoramic radiographs were evaluated with regard to the location and symmetry of the mental foramina in male and female subjects. The results were analyzed using Pearson's $\chi^{2}$ and Fisher's exact test.

Results: Of the 794 panoramic radiographs, 377 met the inclusion criteria for at least one side. The mental foramen was located just below the apex of the second premolar in $62.7 \%$ of the patients and between the first and second premolars in $30 \%$. It was symmetrically located in $79 \%$. No statistically significant differences were found between males and females in the position and symmetry of the mental foramen.

Conclusion: The most common position for the mental foramen in this sample was in line with the second premolar. (Imaging Sci Dent 2013; 43: 71-5)

KEY WORDS: Mandibular Nerve; Radiography, Panoramic; Morocco; Population

\section{Introduction}

The mental nerve is a terminal branch of the inferior alveolar nerve that passes through the mental foramen, supplying sensory innervation to the lower lip, buccal vestibule, and gingiva mesial to the first mandibular molar. ${ }^{1}$ The mental foramen has been reported to vary in position in different ethnic groups. ${ }^{1-11}$ The mental bundle can be traumatized during surgical procedures, such as periapical surgery, extraction of impacted teeth, enucleation of cyst or tumor, and so on, resulting in paresthesia or anesthesia in the area innervated by the nerve. Knowing the site of the mental foramen allows for accurate delivery of local anesthesia of terminal incisive branches of the inferior alveolar nerve. ${ }^{2}$ It also aids in interpreting anatomical landmarks in oral pathology and forensics. ${ }^{5}$

Received May 29, 2012; Revised December 17, 2012; Accepted January 7, 2013 Correspondence to : Dr. Ahmed Chkoura

Department of Oral Surgery, Faculty of Dentistry, University of Mohammed V Souissi, BP 6212, Madinat Al Irfan, Rabat, Morocco

Tel) 212-667-879-951, Fax) 212-537-779-557, E-mail) ahmedchkoura316@ hotmail.com
Although it is often possible to identify the mental foramen radiographically, knowing the normal range of possible locations is essential. The location of the mental foramen has been studied in different populations. ${ }^{1-11}$

Studies by Moiseiwtsch in a North American white population, ${ }^{1}$ Fishel et al, ${ }^{12}$ and Olasoji et al in Northern Nigerian adults ${ }^{9}$ have shown that the most common location of the mental foramen to be between the first and second premolars. However, studies performed in other populations such as Malays, ${ }^{5}$ Asian Indians, ${ }^{5}$ Kenyan Africans, ${ }^{8}$ and Saudis ${ }^{7}$ have indicated that the mental foramen was most commonly positioned in line with the second premolar tooth. The purpose of this study was to investigate the position of the mental foramen in a Moroccan population.

\section{Materials and Methods}

The survey was a descriptive cross-sectional study to identify the position of the mental foramen in relation to the apices of the teeth in panoramic radiographs. A total 
of 794 panoramic radiographs examined in this study were taken by the same practitioner and a single panoramic radiographic machine (Orthophos XG5, Sirona Den-

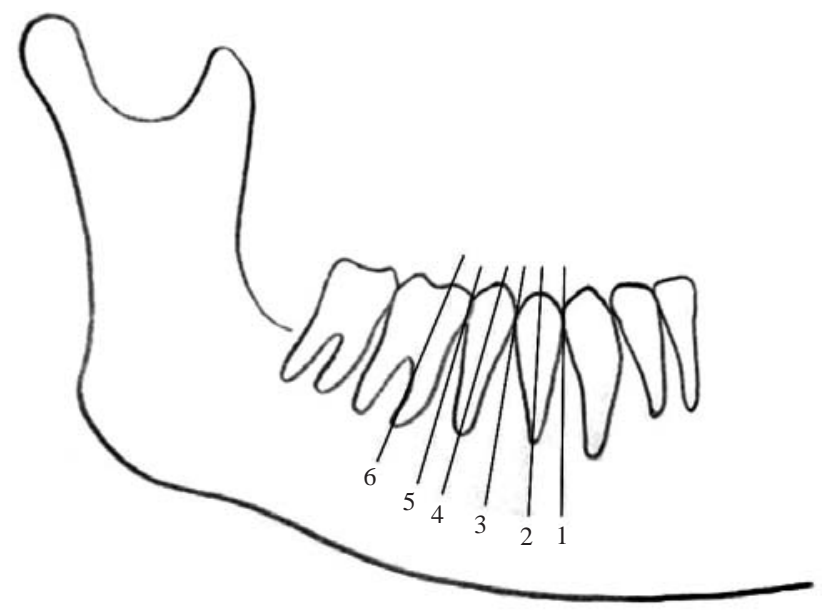

Fig. 1. Schematic representation of the numeric expression of the mental foramen position relative to the teeth. tal Systems GmbH, Bensheim, Germany) from December 2008 to August 2010 in a private dental office in Marrakech, Morocco. Of the 794 panoramic radiographs, 377 met the inclusion criteria for at least one side. Six hundred two mental foramina were evaluated (292 on the right side and 310 on the left side). The age of the patients ranged from 18.2 to 63.9 years.

The exclusion criteria were non-visualization of the mental foramen, presence of missing teeth between the two first mandibular molars, incomplete eruptions of permanent teeth, the presence of severe crowding or spacing between the two first mandibular molars, the presence of radiolucent lesions between the first mandibular molars on both sides, a history of orthodontic treatment, and being under 18 years of age.

The mental foramen was located as a radiolucency that could be traced by following the mandibular canal. The method of evaluation was adopted from Green, ${ }^{3}$ who related the position of the foramen to the teeth, and Ngeow
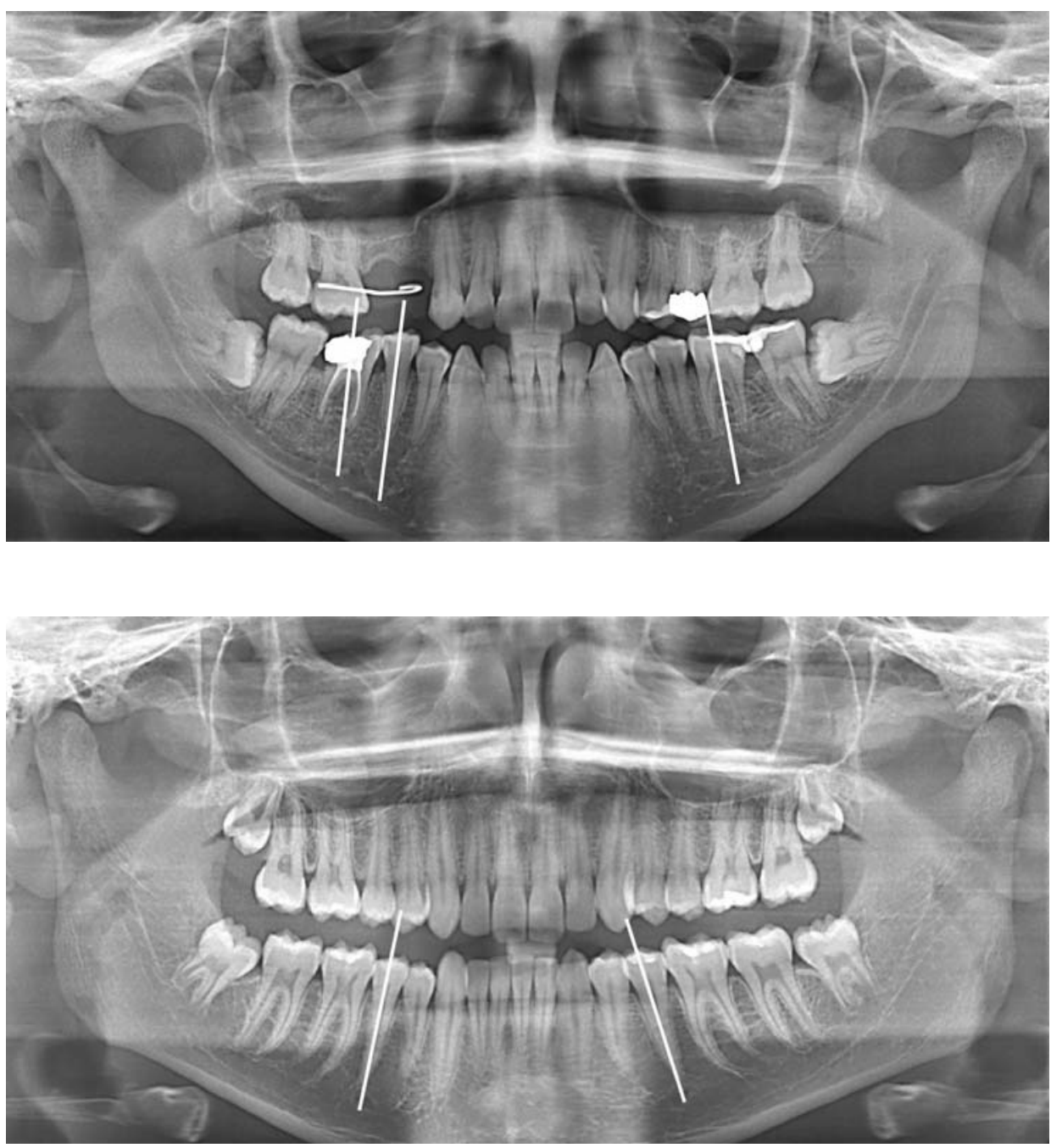

Fig. 2. Mental foramen in position 5 between the apices of the second premolar and the first molar mesial root on the right side and in position 6 at the mesial half of the first molar on the left side.

Fig. 3. Mental foramen in position 4 at the apex of the second premolar on both sides. 


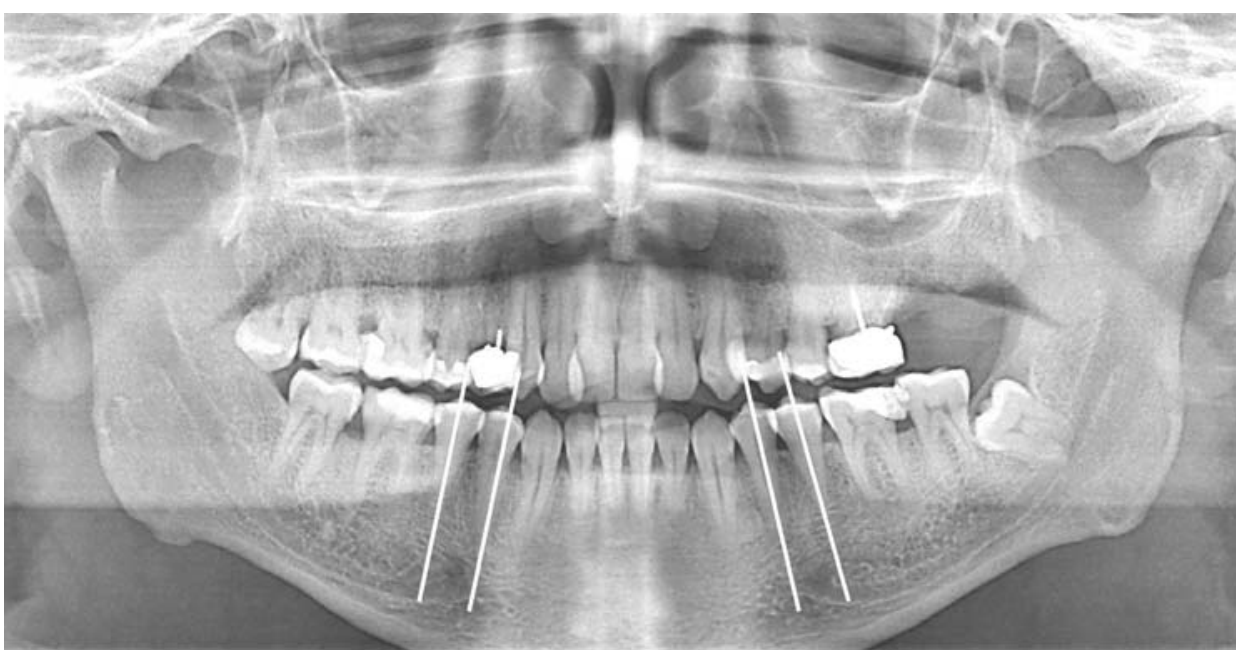

Fig. 4. Mental foramen in position 3 between the apices of the first and second premolar on both sides.

Table 1. Position of the mental foramen in the present study

\begin{tabular}{cccccccc}
\hline Sides & 1 & 2 & 3 & 4 & 5 & 6 & Median position \\
\hline Right (292) & 1 & 3 & 87 & 182 & 17 & 2 & 4 \\
& $(0.3 \%)$ & $(1.0 \%)$ & $(29.8 \%)$ & $(62.3 \%)$ & $(5.8 \%)$ & $(0.7 \%)$ & 3 \\
Left (310) & 2 & 5 & 94 & 196 & 10 & $(1.0 \%)$ & 4 \\
& $(0.6 \%)$ & $(1.6 \%)$ & $(30.3 \%)$ & $(63.3 \%)$ & $(3.2 \%)$ & $(1.0)$ \\
\hline
\end{tabular}

Position of the mental foramen: 1. anterior to the apex of the first premolar, 2. at the apex of the first premolar, 3. between the apices of the first and second premolar, 4. at the apex of the second premolar, 5. between the apices of the second premolar and the first molar, 6 . at the mesial half of the first molar

Table 2. Symmetry of mental foramina according to gender in the present study

\begin{tabular}{lrr}
\hline Gender & Symmetry & Asymmetry \\
\hline Male & $77(78.6 \%)$ & $21(21.4 \%)$ \\
Female & $100(79.4 \%)$ & $26(20.6 \%)$ \\
\hline Total & $177(79.0 \%)$ & $47(21.0 \%)$ \\
\hline
\end{tabular}

and Yuzawati. ${ }^{4}$ All of the radiograph were assessed on a standard radiographic light view box. The edge of a ruler was used to identify the longitudinal axis of the nearest tooth, and the position of the mental foramen was recorded relative to the axis.

The position of the foramen was recorded relative to the adjacent mandibular teeth as follows: (1) anterior to the apex of the first premolar, (2) at the apex of the first premolar, (3) between the apices of the first and second premolar, (4) at the apex of the second premolar, (5) between the apices of the second premolar and the first molar, or (6) at the mesial half of the first molar (Figs. 14).

After evaluation of each panoramic radiograph, the position of the mental foramen on each side was recorded. The location of the mental foramen was reported on the basis of gender and symmetry or asymmetry. In this study symmetry means that the mandibular foramina on both sides have the same anteroposterior position relative to the teeth. Data were then inputted to SPSS 10.0 software (SPSS Inc., Chicago, IL, USA) and Pearson's $\chi^{2}$ and Fisher's exact tests were applied to determine the associations. $\mathrm{p}<0.05$ was taken to indicate statistical significance.

\section{Results}

Of the 377 panoramic radiographs, 161 were of males and 216 of females. The most common position of the mental foramen in this study group was in line with the longitudinal axis of the second premolar (position 4) for both genders ( $65.6 \%$ for males and $60.6 \%$ for females) and both sides $(62.3 \%$ for the right side and $63.3 \%$ for the left side), followed by position 3 (in line with the longitudinal axis of the second premolar) (26.2\% for males and $32.9 \%$ for females; $29.8 \%$ for the right side and $30.3 \%$ for the left side) (Table 1).

The mental foramina were symmetrically located in $79 \%$ of cases (Table 2). No statistically significant differences were seen between males and females in symmetry and asymmetry of the mental foramen position $(\mathrm{p}>0.05)$. 


\section{Discussion}

Knowing the site of the mental foramen allows for accurate delivery of local anesthesia for dental procedures and the avoidance of damage to the nerve in surgical procedures. It also aids in interpreting anatomical landmarks in oral pathology and forensics. ${ }^{5}$

In our series of 377 panoramic radiographs, the most common position for the mental foramen was in line with the second premolar in $62.7 \%$, followed by between the first and second premolars in $30 \%$; thus these two positions accounted for $92.7 \%$ of the cases.

Our results were in accordance with previous studies in other populations showing that the most common location for the mental foramen is in a position aligned with the second premolar, followed by a position between the first and second mandibular premolars. ${ }^{4,6,8,13-16}$ However, in other populations, the mental foramen was most commonly positioned between the first and second premolar tooth. ${ }^{1,2,7,9,12}$ Koppe compared the position of the mental foramen in three populations (Chinese, European, and Indian), and found that it was in line with the axis of the second premolar in the Chinese population and between the first and second premolar in the European and Indian populations. ${ }^{10}$ According to our study, the mental foramina were usually symmetrically located on both sides. Oguz and Bozkir studied the location of mental foramina in dried human mandibles of Turkish young adult males. The authors demonstrated that the mental foramen was located below the root of the second premolar in $61.76 \%$ on the right sides and $50 \%$ on the left sides of the mandibles, and the foramen was present between the roots of the first and second premolars in $38.2 \%$ on the right and in $50 \%$ on the left. ${ }^{17}$ Mbajiorgu et al demonstrated that the position percentage of the mental foramen was highest below the lower second premolar tooth on the right side and posterior to it on the left side among black Zimbabweans. ${ }^{11}$

Panoramic radiographs were used in this study because the mental foramen was seen more consistently on the wide field of view in panoramic radiographs of the mandible than on periapical radiographs. ${ }^{2}$ Philips et al demonstrated that the size of the foramen on panoramic radiographs was slightly larger than that reported on periapical radiographs, but they stated that the horizontal position of the mental foramen on panoramic radiographs generally agreed with the position reported on periapical radiographs. ${ }^{18}$

The weakness of our study is the use of panoramic radiographs for localization of the mental foramen instead of the use of an anatomic study on skulls, given that Philips et $\mathrm{al}^{18}$ stated that panoramic radiographs showed a greater displacement of the foramen compared with anatomical measurements; however, the difference in the horizontal position found by the two methods was not statistically significant. Further anatomical studies using skulls should be conducted in a Moroccan population.

\section{Acknowledgment}

The authors wish to thank Dr. Benmalek Mehdi for his data used in this study.

\section{References}

1. Moiseiwitsch JR. Position of the mental foramen in a North American, white population. Oral Surg Oral Med Oral Pathol Oral Radiol Endod 1998; 85: 457-60.

2. Haghanifar S, Rokouei M. Radiographic evaluation of the mental foramen in a selected Iranian population. Indian J Dent Res 2009; 20: 150-2.

3. Green RM. The position of the mental foramen: a comparison between the southern (Hong Kong) Chinese and other ethnic and racial groups. Oral Surg Oral Med Oral Pathol 1987; 63: 287-90.

4. Ngeow WC, Yuzawati Y. The location of the mental foramen in a selected Malay population. J Oral Sci 2003; 45: 171-5.

5. Neo J. Position of the mental foramen in Singaporean Malays and Indians. Anesth Prog 1989; 36: 276-8.

6. Mwaniki DL, Hassanali J. The position of mandibular and mental foramina in Kenyan African mandibles. East Afr Med J 1992; 69: 210-3.

7. al Jasser NM, Nwoku AL. Radiographic study of the mental foramen in a selected Saudi population. Dentomaxillofac Radiol 1998; 27: 341-3.

8. Sankar DK, Bhanu SP, Susan PJ. Morphometrical and morphological study of mental foramen in dry dentulous mandibles of South Andhra population of India. Indian J Dent Res 2011; 22: 542-6.

9. Olasoji HO, Tahir A, Ekanem AU, Abubakar AA. Radiographic and anatomic locations of mental foramen in northern Nigerian adults. Niger Postgrad Med J 2004; 11: 230-3.

10. Koppe T. Summary of: A comparative anthropometric study of the position of the mental foramen in three populations. $\mathrm{Br}$ Dent J 2012; 212: 188-9.

11. Mbajiorgu EF, Mawera G, Asala SA, Zivanovic S. Position of the mental foramen in adult black Zimbabwean mandibles: a clinical anatomical study. Cent Afr J Med 1998; 44: 24-30.

12. Fishel D, Buchner A, Hershkowith A, Kaffe I. Roentgenologic study of the mental foramen. Oral Surg Oral Med Oral Pathol 1976; 41: 682-6.

13. Miller JA Jr. Studies on the location of the lingula, mandibular foramen and mental foramen. Anat Rec 1953; 115: 349.

14. Phillips JL, Weller RN, Kulild JC. The mental foramen: 1. Size, 
orientation, and positional relationship to the mandibular second premolar. J Endod 1990; 16: 221-3.

15. Matsuda Y. Location of the dental foramina in human skulls from statistical observations. Int J Orthod Oral Surg Radiogr 1927; 13: 299-305.

16. Tebo HG, Telford IR. An analysis of the variations in position of the mental foramen. Anat Rec 1950; 107: 61-6.
17. Oguz O, Bozkir MG. Evaluation of location of mandibular and mental foramina in dry, young, adult human male, dentulous mandibles. West Indian Med J 2002; 51: 14-6.

18. Phillips JL, Weller RN, Kulild JC. The mental foramen: 3. Size and position on panoramic radiographs. J Endod 1992; 18: 383-6. 